\title{
Wind farm reactive power optimization by using imperialist competitive algorithm
}

\begin{abstract}
In this paper a new evolutionary computing method based on imperialist competitive algorithm (ICA) is used for optimization of the reactive power in a wind farm. The output power and also the reactive power of wind farms are not constant due to the oscillation in wind speed. Reactive power optimization is known as an efficient way to have an improvement in power quality and also to reduce power loss. The conventional optimization algorithms have some drawbacks, such as slow convergence and premature. ICA as one of the newest optimization algorithm could be applied in order to optimization of the reactive power and overcomes the difficulties which are coming from the traditional methods. In this paper, the reactive power consumption of a wind turbine is optimized by using (ICA) method. To illustrate the application of the method, a wind farm with some uncertainties is provided. Finally the results of the ICA method are compared with the one of conventional method. Results show that the proposed reactive power optimization method is simple and effective.
\end{abstract}

Keyword: Evolutionary computing; Imperialist competitive algorith; Reactive power optimization; Renewable energy; Wind farm 